ARTICLE

DOI: $10.1038 / s 41467-018-07194-x$

\title{
Samarium(II) folding cascades involving hydrogen atom transfer for the synthesis of complex polycycles
}

Mateusz P. Plesniak' ${ }^{1}$ Monserrat H. Garduño-Castro ${ }^{1}$, Philipp Lenz ${ }^{1}$, Xavier Just-Baringo ${ }^{1}$ \& David J. Procter (i) ${ }^{1}$

The expedient assembly of complex, natural product-like small molecules can deliver new chemical entities with the potential to interact with biological systems and inspire the development of new drugs and probes for biology. Diversity-oriented synthesis is a particularly attractive strategy for the delivery of complex molecules in which the 3-dimensional architecture varies across the collection. Here we describe a folding cascade approach to complex polycyclic systems bearing multiple stereocentres mediated by reductive single electron transfer (SET) from $\mathrm{Sml}_{2}$. Simple, linear substrates undergo three different folding pathways triggered by reductive SET. Two of the radical cascade pathways involve the activation and functionalization of otherwise inert secondary alkyl and benzylic groups by 1,5hydrogen atom transfer (HAT). Combination of $\mathrm{Sml}_{2}$, a privileged reagent for cascade reactions, and 1,5-HAT can lead to complexity-generating radical sequences that unlock access to diverse structures not readily accessible by other means.

\footnotetext{
${ }^{1}$ School of Chemistry, University of Manchester, Oxford Road, Manchester M13 9PL, UK. Correspondence and requests for materials should be addressed to D.J.P. (email: david.j.procter@manchester.ac.uk)
} 
T he synthesis of complex small molecules that can interact with biological systems is of paramount importance in science. Advances in the field can lead to new therapeutic agents and probes for molecular biology that allow intricate biological processes to be unravelled ${ }^{1,2}$. Target-oriented synthesis, medicinal and combinatorial chemistry, and diversity-oriented synthesis are the main strategies used to gain access to collections of complex small molecules ${ }^{3}$. While the first two approaches target defined structure space of known biological relevance, diversity-oriented synthesis attempts to deliver a diverse range of molecular architectures than can give access to unexplored activity and the promise of greater potency ${ }^{4-9}$. These products

a

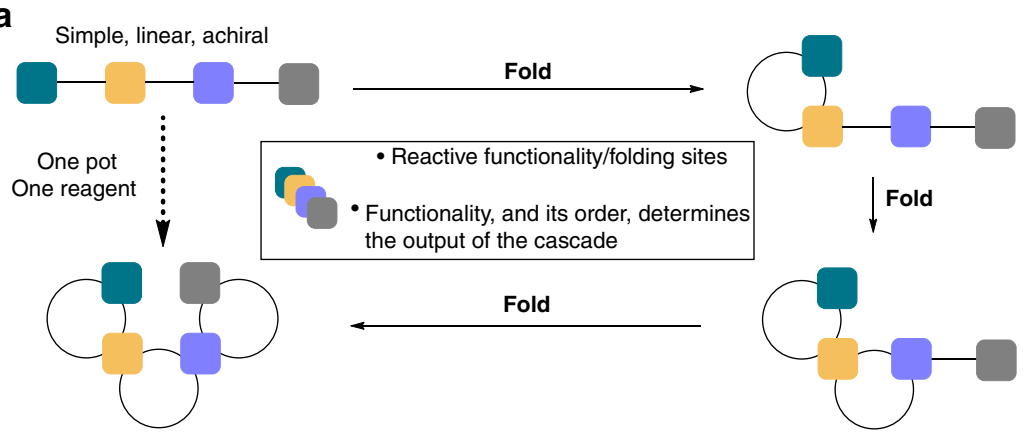

Complex, 3D, chiral

b

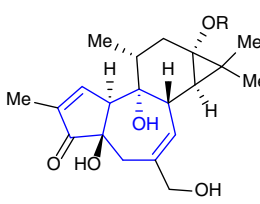

$\mathrm{R}=\mathrm{Ac}$, prostratin

$\mathrm{R}=\mathrm{COBn}$, DPP

- Active against latent HIV
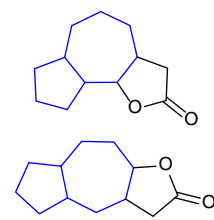

Sesquiterpene lactones

- Anticancer

- Anti-inflammatory

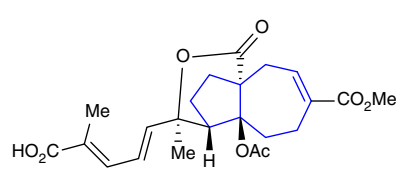

Pseudolaric acid B

- Novel mode of antimitotic activity

- Active against multi-resistant cancers

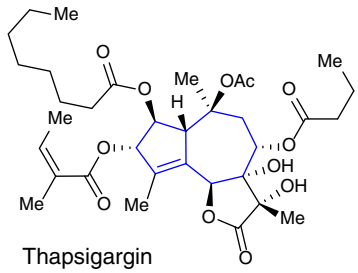

- SERCA-pump protein inhibitor

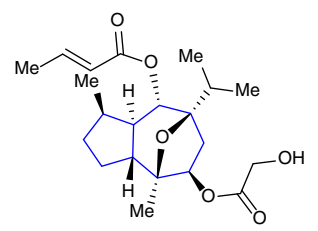

Englerin A

- Anticancer

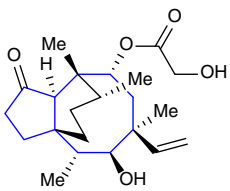

Pleuromutilin

- Antibiotic
C

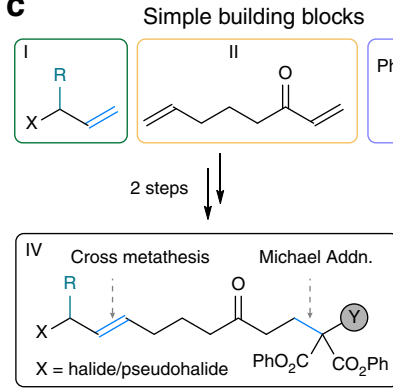

Skeletal diversity determining groups
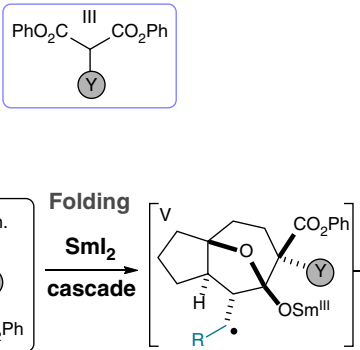

Key radical intermediates

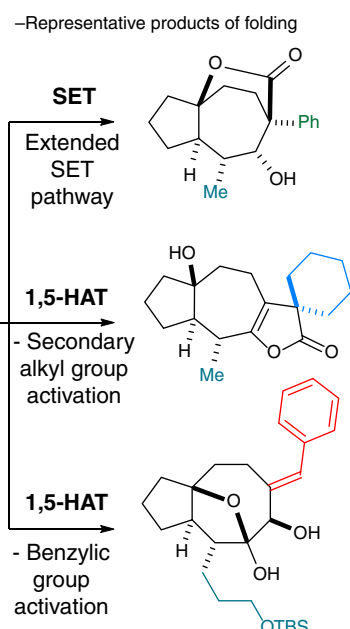

Fig. 1 A folding cascade approach that delivers polycyclic architectures and the samarium(II) folding cascade approach. The folding cascade approach, examples of biologically active natural products bearing [5,7] and [5,8] fused bicyclic motifs, and a samarium(II)-mediated, one-pot cascade reaction enabling access to these architectures, are shown. a Folding cascade approaches convert simple, linear starting materials to complex 3D polycyclic architectures through the sequential action of a single reagent on functional groups arranged in the substrate to be folded. $\mathbf{b}$ Biologically relevant natural products possessing multiple stereocentres and sites of oxygenation, and $[5,7]$ or $[5,8]$ ring systems include prostratin, pseudolaric acid B, englerin A, sesquiterpene lactones such as thapsigargin, and pleuromutilin. c This work involves the development of a one-pot approach to complex polycyclic architectures from simple starting materials. The approach exploits samarium(II) folding cascades featuring 1,5-HAT functionalisation of tertiary and benzylic $\mathrm{C}-\mathrm{H}$ bonds 
often possess unique three-dimensional (3D) shapes ${ }^{10-14}$ that can enhance their interaction with biological targets, in contrast to more traditional medicinal chemistry scaffolds that are two dimensional ${ }^{15-17}$.

A main goal of diversity-oriented approaches is to concomitantly access structurally complex molecules and skeletal diversity ${ }^{18}$. In this regard, cascade processes that convert simple starting materials into complex products, in one pot, are ideal ${ }^{19-}$ 22 . Furthermore, approaches in which the presence of a particular group in a substrate can direct the reaction down one of several pathways, the so-called substrate-based approaches, are highly desirable tools for accessing skeletal diversity ${ }^{23}$.

Recent studies in the field of radical chemistry have shown the great potential of open shell intermediates, generated selectively under mild conditions, for use in cascade processes in which complex polycyclic systems bearing multiple stereocentres are formed in a single operation ${ }^{24-26}$. Of particular interest are radical processes that exploit hydrogen atom transfer (HAT) to functionalise remote, inert $\mathrm{C}-\mathrm{H}$ bonds ${ }^{27-29}$. In the context of complexity generation, HAT processes allow radicals to be relocated to sites at which new bond-forming events can take place, thus extending reaction cascades ${ }^{30}$. While recent reports in the field of diversity-oriented synthesis have utilised radical reactions to access diverse molecular space ${ }^{31-34}$, the exploitation of HAT in such processes remains largely unexplored.

Herein we present a folding cascade approach in which simple, linear starting materials are converted to complex 3D polycyclic architectures through the sequential action of a single reagent on functional groups arranged in the substrate to be folded (Fig. 1a). ${ }^{18-20}$ In particular, using the reductive single electron transfer (SET) reagent samarium diiodide $\left(\mathrm{SmI}_{2}\right)^{35-38}$ in folding cascades, radical (and anionic) character is generated at various points during the one-pot/one-reagent folding process and utilised for selective carbon-carbon bond formation. In our substrate-based approach, skeletal diversity is achieved by the formation of different polycyclic ring systems, quaternary centres, and spirocyclic functionalities, accompanied by the generation of up to five stereocentres. The four distinct scaffolds generated resemble those found in many complex and biologically active natural products (Fig. 1 b). The starting materials IV to be folded are typically accessed in modular fashion from terminal alkenes I bearing allylic functionality $\mathrm{X}$, keto diene building block II, and diphenoxy malonates III, in two steps (Michael addition and alkene cross-metathesis). In particular, malonates III contain groups that determine the course of the folding cascades. Depending on the nature of the group Y, key radical intermediates $\mathbf{V}$ can follow three distinct pathways to generate four types of complex scaffold. Two of the folding pathways involve 1,5-hydrogen atom transfer (HAT), an important process that has rarely been exploited in the chemistry of $\mathrm{SmI}_{2}{ }^{39-41}$ or in diversity-oriented synthesis (Fig. 1c).

\section{Results}

Samarium(II) folding cascades involving an extended SET pathway. The mechanistic platform upon which our $\mathrm{SmI}_{2}$ mediated folding radical cascades are built involves a Barbier cyclisation-lactonisation-lactone radical cyclisation sequence to generate key radical intermediates $\mathbf{V}$ with high diastereocontrol (Fig. 2). Allyl samariums 1 are initially formed by reduction of the allylic halide/pseudohalide moiety $\left(\mathrm{X}=\mathrm{Br}\right.$ or $\mathrm{OC}(\mathrm{O}) \mathrm{C}_{6} \mathrm{H}_{4}-4$ $\mathrm{CF}_{3}$ ) and undergo diastereoselective addition to the ketone carbonyl to give samarium alkoxides 2. Diastereoselective lactonisation via transition structure $\mathbf{3}$ establishes a $\mathbf{a}$-all-carbon quaternary stereocentre and gives lactones 4 . The addition of a protic additive to the reaction vessel activates $\mathrm{SmI}_{2}{ }^{42,43}$ and switches on the next stage of the sequence: SET reduction of the lactone carbonyl in $\mathbf{4}$ gives ketyl radicals $\mathbf{5}$ and 5-exo-trig radical cyclisation generates the key radicals $\mathbf{V}^{44}$.

Initially, substrates were designed to follow an extended SET pathway in which radicals $\mathbf{V}$ were reduced further and the anions protonated by the $\mathrm{H}_{2} \mathrm{O}$ additive (Fig. 3a). Subsequent opening of the hemiketal in the products and SET reduction of the resulting ketones gave secondary alcohols 7a'-c' and 7a-7c from the folding of simple substrates with complete diastereocontrol (Fig. 3b). Alternatively, the ketyl radical intermediates formed in the ketone reduction can be trapped by a pendant heteroarene to forge spirocyclic scaffold 7d' (Fig. 3c). Notably, linear starting materials $\mathbf{6 a}-\mathbf{6 c}$, prepared from diphenylmalonate building blocks III, underwent lactonisation in the final stage of the cascade process to deliver bridged lactone products $7 \mathbf{a}-7 \mathbf{c}$. All products from this extended SET pathway were obtained in moderate to
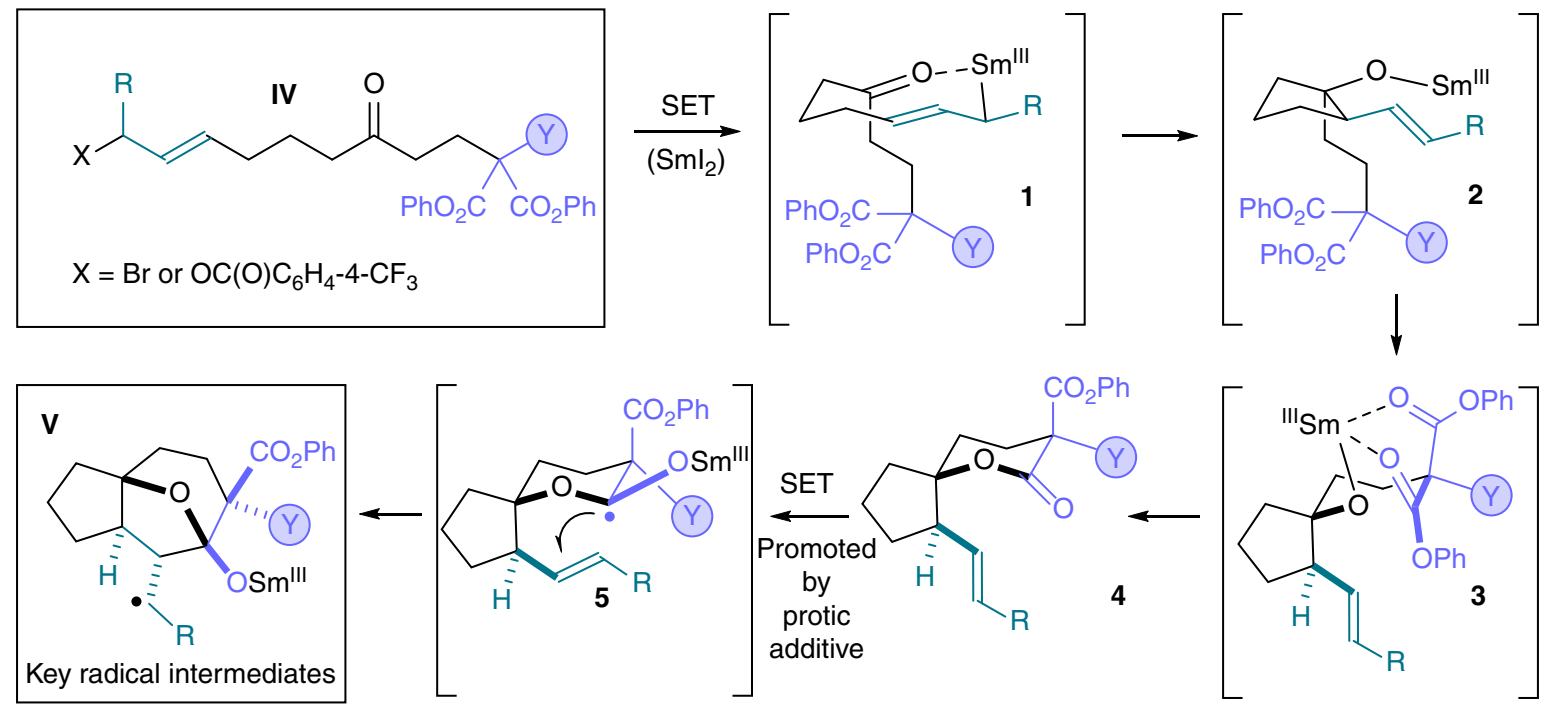

Fig. 2 Mechanistic platform for the radical folding cascades. A mechanism for the common stages of the samarium(II) folding cascades and the formation of the key radical intermediates $\mathbf{V}$ is shown. The sequence commences with an intramolecular, samarium(II)-mediated Barbier reaction to give alkoxides $\mathbf{2}$ which yield $\mathbf{4}$ after diastereoselective lactonisation. Addition of water then triggers lactone radical cyclisations involving radicals $\mathbf{5}$ 
good overall isolated yield (30-73\%) and as single diastereoisomers (with the exception of $\mathbf{7 d}$ '). Notably, up to 5 contiguous stereocentres and 3 rings are formed in the folding cascades.

Samarium(II) folding cascades involving 1,5-HAT from a secondary centre. To extend the radical cascades and to functionalise otherwise unreactive substrate sites during folding, we sought to relocate the radicals in $\mathbf{V}$ using intramolecular HAT, and to trap the new radicals, thus accessing diverse structures. Crucially, HAT processes have little precedent in the chemistry of $\mathrm{SmI}_{2}$ as radical intermediates are typically reduced rapidly to the corresponding carbanions ${ }^{45}$. We reasoned that careful substrate design could lead to radical intermediates $\mathbf{V}$ in which the radical centre was sufficiently close to the site of abstraction for the rate of 1,5-HAT to outcompete the usual radical reduction and protonation that dominates $\mathrm{SmI}_{2}$ chemistry. The proposed 1,5-HAT pathway was explored using malonate components III bearing secondary alkyl groups possessing tertiary $\mathrm{C}-\mathrm{H}$ bonds $\beta$ - to the ester carbonyls. Pleasingly, simple substrates $\mathbf{6 d - j}$ underwent radical folding cascades to give complex tricyclic lactones $\mathbf{7 d - j}$ in good overall yield and as single diastereoisomers (Fig. 4a). Notably, malonate substrates $\mathbf{6 e - h}$ containing carbo- and heterocyclic motifs, conveniently introduced using a suitably substituted malonate component III, delivered lactone products bearing carbo- and hetero-spirocyclic rings $\mathbf{7 e - h}$.

The mechanism of the folding cascades involving 1,5-HAT from a secondary alkyl group is shown in Fig. 4 b. After the initial Barbier cyclisation - lactonisation - lactone cyclisation sequence (see Fig. 2), 1,5-HAT in radical 8 activates the secondary alkyl group in the malonate unit and radicals $\mathbf{9}$ are formed. Crucially, reduction of tertiary radicals $\mathbf{9}$ to the corresponding anions is slower than intramolecular radical addition and the radicals undergo 1,2-migration of the ester group ${ }^{46}$ to give stabilised radicals 11 after hemiketal collapse. Subsequent SET gives samarium enolates $12^{47}$ that undergo lactonisation. As proposed, the facile nature of the 1,5-HAT process, relative to reduction of $\mathbf{8}$ to the corresponding anions, is likely the result of the proximity

a<smiles>[X]C([R])C=CCCCC(=O)CCCC(=O)Oc1ccccc1</smiles>
$\mathrm{X}=\mathrm{Br}, \mathrm{X}=\mathrm{OC}(\mathrm{O}) \mathrm{C}_{6} \mathrm{H}_{4}-4-\mathrm{CF}_{3}$ $\mathrm{PhO}_{2} \mathrm{C} \quad \mathrm{CO}_{2} \mathrm{Ph}$<smiles>C[C@@H]1[C@@H]2CCC[C@]2(O)CCC[C@H]1O</smiles>

7a' $59 \%$ X-ray $\mathrm{R}^{1}=\mathrm{H}$

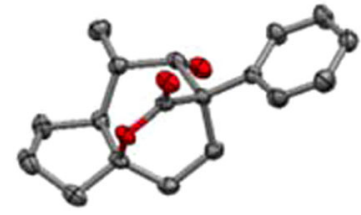

X-Ray of $7 a$<smiles>CCC[SeH2]CCCC[C@@H]1[C@@H]2CCC[C@]2(O)CCC[C@H]1O</smiles>
$7 \mathbf{b}^{\prime} 55 \%^{a}$
$\mathrm{R}^{1}=-\left(\mathrm{CH}_{2}\right)_{3}$ OTBS TRS<smiles>O[C@H]1CCC[C@]2(O)CCC[C@@H]2[C@H]1CCCc1ccccc1</smiles>
$7 c^{\prime} 44 \% \%^{a}$
$\mathrm{R}^{1}=-\mathrm{CH}_{2} \mathrm{CH}_{2} \mathrm{Ph}$<smiles>C[C@@H]1[C@@H]2CCC[C@]23CC[C@](c2ccccc2)(C(=O)O3)[C@@H]1O</smiles>
$7 a 39 \%$ X-ray
$R^{1}=H, R^{2}=P h$

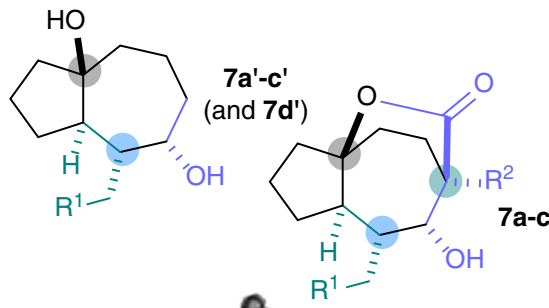
7a-c $R^{1}$ 二

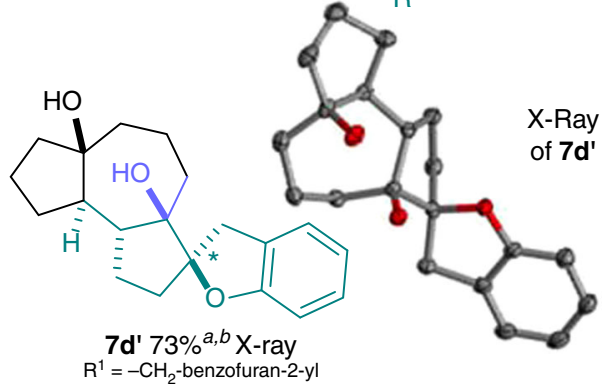<smiles>CC[C@]12CCC3(CCC[C@H]3[C@H](C)[C@@H]1O)OC2=O</smiles>

7b 30\%

$R^{1}=H, R^{2}=E$<smiles>C[C@H]1[C@@H](O)[C@@H]2CCC[C@]23CC[C@]1(C)C(=O)O3</smiles>

7c $45 \%$

$R^{1}=H, R^{2}=M e$

${ }^{a} \mathrm{X}=\mathrm{OC}(\mathrm{O}) \mathrm{C}_{6} \mathrm{H}_{4}-4-\mathrm{CF}_{3}$ and 14 equiv. of $\mathrm{Sml}_{2}$ were used. ${ }^{b_{1}: 1.3}$ mixture of diastereoisomers at the highlighted center.

b

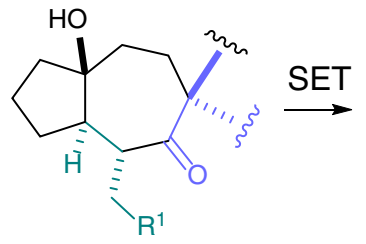<smiles>C[C@@H]1C(O)C(C)(C)CCC2(O)CCCC12</smiles>

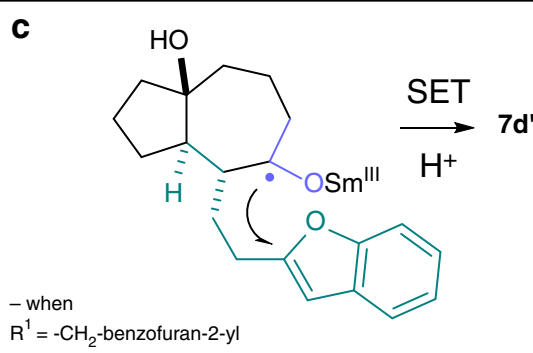

Fig. 3 Folding radical cascades following extended SET pathways. Samarium(II) folding cascades involving an extended SET pathway from key radical intermediates $\mathbf{V}$ are shown. a Folding of the substrates $\mathbf{6} \mathbf{a}^{\prime}-\mathbf{d}^{\prime}$ allowed access to diols $\mathbf{7} \mathbf{a}^{\prime}-\mathbf{d}$ ' bearing up to five new stereocentres. The use of malonatederived substrates $\mathbf{6 a}-\mathbf{6} \mathbf{c}$ allowed the synthesis of complex lactones $\mathbf{7 a - 7} \mathbf{c}$. $\mathbf{b}$ The formation of products involves SET reduction of the carbonyl and diastereoselective protonation to give diols $\mathbf{7} \mathbf{a}^{\prime}-\mathbf{c}^{\prime}$. In the case of products $\mathbf{7 a - c}$, the alcohol formed in the last step undergoes lactonisation. $\mathbf{c}$ The formation of product $\mathbf{7 d}$ ' involves a dearomatising radical cyclisation of the samarium(II) ketyl radical intermediate formed upon ketone reduction 
of the radicals in $\mathbf{V}$, formed from the diastereoselective lactonisation-lactone cyclisation sequence, to the alkyl sidechain located $\alpha$ to the ester (Fig. $4 \mathrm{~b}$ ).

Samarium(II) folding cascades involving 1,5-HAT from a benzylic centre. Building on the successful use of 1,5-HAT to activate secondary alkyl groups in the folding cascades, we proposed that remote activation of a benzylic position could lead to alternative product architectures. Benzyl-containing substrates $\mathbf{6 k}-\mathbf{q}$ were prepared using suitably substituted malonates III in two steps. Exposure of $\mathbf{6 k - q}$ to $\mathrm{SmI}_{2}$ delivered 5,8-carbocylic cascade products $\mathbf{7 k}-\mathbf{q}$ as single diastereoisomers in moderate yield. Notably, the folding cascades feature five bond-forming events and establish five new contiguous stereocentres. In this case it was found that $\mathrm{MeOH}$, and not $\mathrm{H}_{2} \mathrm{O}$, was the optimal protic additive for use in conjunction with $\mathrm{SmI}_{2}{ }^{48-50}$. This may be due to the particular conformation of lactone intermediates 4 (Fig. 2; when $\mathrm{Y}=\mathrm{CH}_{2} \mathrm{Ar}$ ) and promotion of lactone carbonyl reduction by coordination of $\mathrm{SmI}_{2}$ to the alpha ester group, thus negating the need for water activation of the reagent. ${ }^{51}$

Importantly, the reductive SET conditions were also shown to be compatible with the presence of halide (formation of $\mathbf{7 l}$ and $\mathbf{7 m}$ ), methoxy (formation of $\mathbf{7 n}$ ), and heteroaryl substituents (formation

a<smiles>[Y]=[W]Br</smiles>

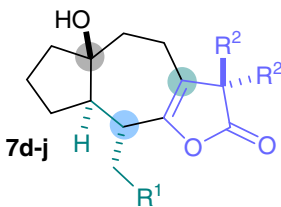<smiles>[Y19][C@H]1C2=C(CC[C@]3(O)CCC[C@@H]13)[C@]([Y4])(C)C(=O)O2</smiles>

7d $60 \%$ X-ray $R^{1}=H, R^{2}=M e$<smiles>C[C@H]1C2=C(CC[C@]3(O)CCC[C@@H]13)C1(CCCCC1)C(=O)O2</smiles>

7f $51 \%$ X-ray $\mathrm{R}^{1}=\mathrm{H}, \mathrm{R}^{2}=-\left(\mathrm{CH}_{2}\right)_{5}-$<smiles>C[C@H]1C2=C(CC[C@]3(O)CCC[C@@H]13)C1(CCOCC1)C(=O)O2</smiles>

7h $51 \%$ $\mathrm{R}^{1}=\mathrm{H}, \mathrm{R}^{2}=-\left(\mathrm{CH}_{2}\right)_{2} \mathrm{O}\left(\mathrm{CH}_{2}\right)_{2}-$

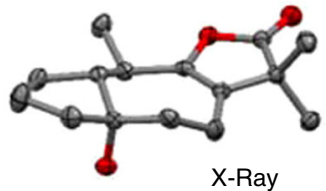

of $7 d$

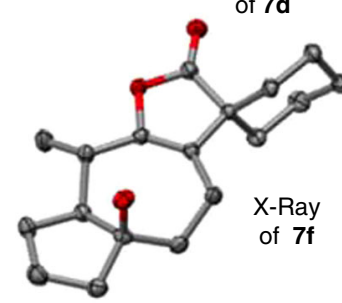

X-Ray
of $7 f$<smiles>CC1(C)C(=O)OC2=C1CC[C@]1(O)CCC[C@H]1[C@H]2CCCc1ccccc1</smiles>

$7 i 56 \%^{a}$

$\mathrm{R}^{1}=-\mathrm{CH}_{2} \mathrm{CH}_{2} \mathrm{Ph}, \mathrm{R}^{2}=\mathrm{Me}$<smiles>C[C@H]1C2=C(CC[C@]3(O)CCC[C@@H]13)C1(CCCC1)C(=O)O2</smiles>

7 e $56 \%$ $\mathrm{R}^{1}=\mathrm{H}, \mathrm{R}^{2}=-\left(\mathrm{CH}_{2}\right)_{4}{ }^{-}$<smiles>C[C@H]1C2=C(CC[C@]3(O)CCC[C@@H]13)C1(CCCCCC1)C(=O)O2</smiles>
$\mathrm{R}^{1}=\mathrm{H}, \mathrm{R}^{2}=-\left(\mathrm{CH}_{2}\right)_{6}-$<smiles>CC1(C)C(=O)OC2=C1CC[C@]1(O)CCC[C@H]1[C@H]2CCCC[SeH3]</smiles>

7j $55 \%^{a}$

$\mathrm{R}^{1}=-\left(\mathrm{CH}_{2}\right)_{3}$ OTBS, $\mathrm{R}^{2}=\mathrm{Me}$

${ }^{a} \mathrm{X}=\mathrm{OC}(\mathrm{O}) \mathrm{C}_{6} \mathrm{H}_{4}-4-\mathrm{CF}_{3}$ and 12 equiv. of $\mathrm{Sml}_{2}$ was used

b

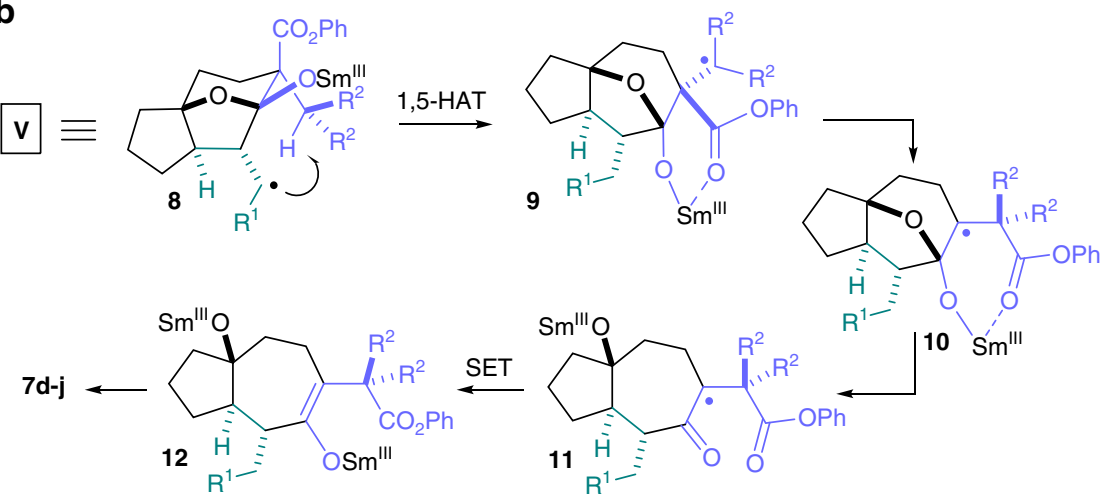

Fig. 4 Folding radical cascades involving 1,5-HAT and activation of a secondary alkyl group. A samarium(II) folding cascade involving 1,5-HAT activation of secondary alkyl groups in key radical intermediates $\mathbf{V}$ is shown. a Scope of the samarium(II) folding cascade involving activation of a secondary alkyl group by 1,5-HAT to give products $\mathbf{7 d} \mathbf{d}-\mathbf{j}$. b The mechanism of folding involves 1,5-HAT in key radical intermediates $\mathbf{V}$ to give $\mathbf{9}$. A 1,2-ester shift then generates radical intermediates $\mathbf{1 0}$ that, after hemiketal opening and SET reduction, give samarium(III) enolates $\mathbf{1 2}$. The final products $\mathbf{7 d} \mathbf{d} \mathbf{j}$ are obtained by lactonisation of enolates $\mathbf{1 2}$ 
of $\mathbf{7 p}$ ). The carbon-halogen bonds in $\mathbf{7 1}$ and $\mathbf{7 m}$ provide useful handles for further functionalisation of the cascade products (Fig. 5a). The mechanism of the folding cascade is set out in Fig. 5b. After the initial Barbier cyclisation-lactonisation-lactone cyclisation sequence (see Fig. 2), 1,5-HAT in key radical intermediates 13 allows activation of the benzylic position to give radicals $\mathbf{1 4}$. In contrast to the pathway involving tertiary radicals $\mathbf{9}$, the benzylic radicals 14 undergo fragmentation, presumably promoted by the formation of an alkene that is in conjugation with the aromatic ring and the ester carbonyl. The resultant ketyltype radicals 15 undergo acyloin-type cyclisation to deliver ketones $\mathbf{1 6}$ that are then diastereoselectively reduced (Fig. 5b).
Mechanistic experiments. Mechanistic experiments involving a labelled additive and substrate have been used to probe the HAT pathways (Fig. 6). We first confirmed that a substrate lacking a substituent capable of HAT, substrate 6a, undergoes cascade cyclisation to give labelled product, $D_{2}-7 \mathbf{a}$, when exposed to $\mathrm{SmI}_{2}$ and $\mathrm{D}_{2} \mathrm{O}$ (rather than $\left.\mathrm{H}_{2} \mathrm{O}\right)^{52}$. This observation is consistent with radicals $\mathbf{V}$ being reduced and the resulting organosamariums protonated when HAT processes are not in operation. Furthermore, when substrate 6d, bearing an iso-propyl substituent capable of undergoing HAT, was exposed to identical $\mathrm{SmI}_{2}$ and $\mathrm{D}_{2} \mathrm{O}$ conditions, no deuterium incorporation in the methyl group of product $\mathbf{7 d}$ was observed. This suggests that the primary radical

a

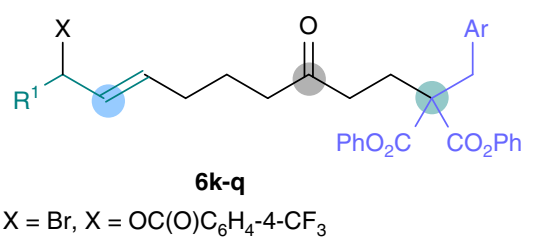

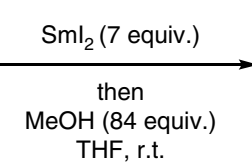

THF, r.t.

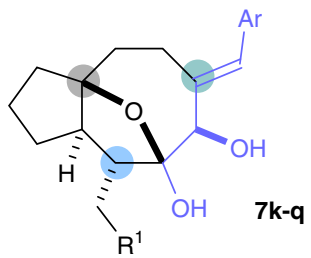

$\mathrm{X}=\mathrm{Br}, \mathrm{X}=\mathrm{OC}(\mathrm{O}) \mathrm{C}_{6} \mathrm{H}_{4}-4-\mathrm{CF}_{3}$<smiles>[Y6][Y16]([H])([H])[H]</smiles>

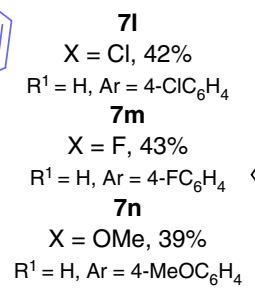

71

$\mathrm{X}=\mathrm{Cl}, 42 \%$ $\mathrm{R}^{1}=\mathrm{H}, \mathrm{Ar}=4-\mathrm{ClC}_{6} \mathrm{H}_{4}$ $7 \mathrm{~m}$ $X=F, 43 \%$ $\mathrm{R}^{1}=\mathrm{H}, \mathrm{Ar}=4-\mathrm{FC}_{6} \mathrm{H}_{4}$

$7 n$ $\mathrm{X}=\mathrm{OMe}, 39 \%$ $\mathrm{R}^{1}=\mathrm{H}, \mathrm{Ar}=4-\mathrm{MeOC}_{6} \mathrm{H}_{4}$

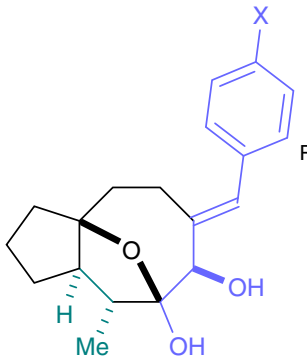

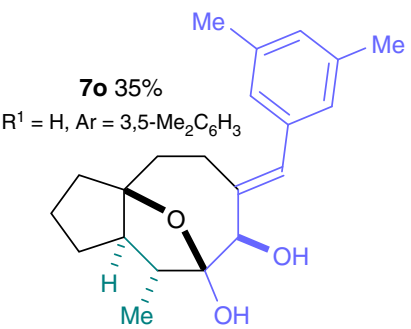
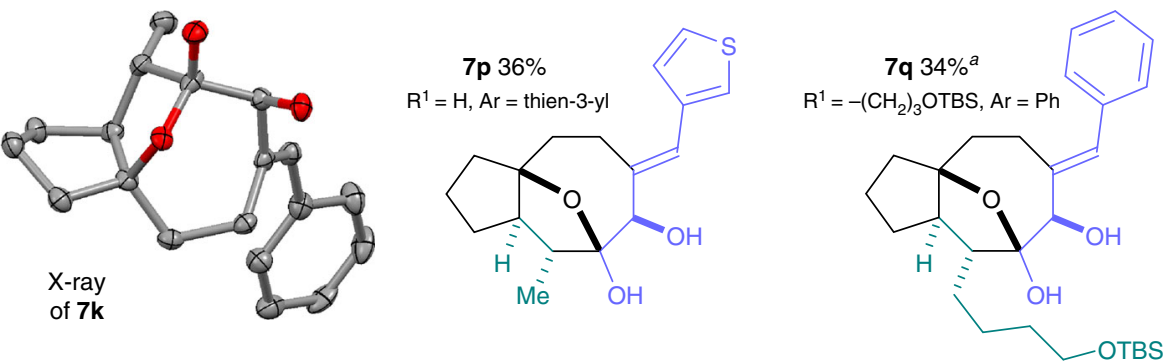

${ }^{a} \mathrm{X}=\mathrm{OC}(\mathrm{O}) \mathrm{C}_{6} \mathrm{H}_{4}-4-\mathrm{CF}_{3}$ and 12 equiv. of $\mathrm{Sml}_{2}$ was used.

b

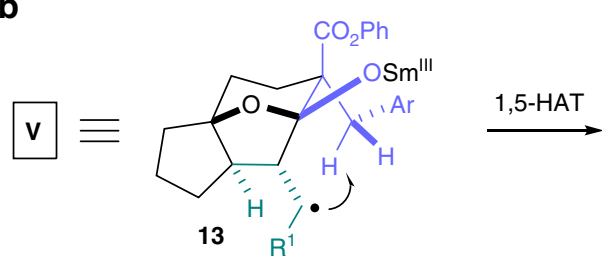

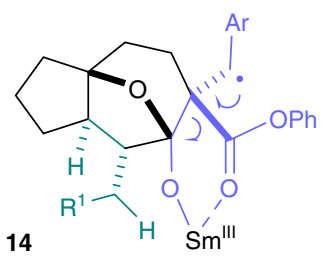<smiles>CCCC</smiles><smiles>[R]C[C@H]1[C@H]2CCC[C@]23CC/C(=C/Br)C(=O)[C@]1(O)O3</smiles>

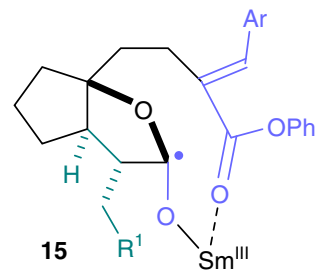

Fig. 5 Folding radical cascades involving 1,5-HAT and activation of a benzylic group. A samarium(II) folding cascade involving activation of benzylic groups by 1,5-HAT in key radical intermediates $\mathbf{V}$ is shown. a The scope of the samarium(II) folding cascades involving benzylic group activation by 1,5-HAT to give products $\mathbf{7 k - q}$. b The mechanism of folding involves 1,5-HAT in key radical intermediates $\mathbf{V}$ to give $\mathbf{1 4}$ and subsequent fragmentation to give ketyl-type radicals $\mathbf{1 5}$. Intramolecular acyloin reaction then delivers $\mathbf{1 6}$ that undergo diastereoselective reduction to give products $\mathbf{7 k - \mathbf { q }}$ 
a<smiles>O=C(CCC/C=C/CBr)CCC(C(=O)O)(C(=O)Oc1ccccc1)c1ccccc1</smiles>

$6 a$<smiles>[M]C([M])(C(=O)Oc1ccccc1)C(CCC(=O)CCCC=CCBr)(C(=O)O)C(=O)O</smiles>

$6 d$

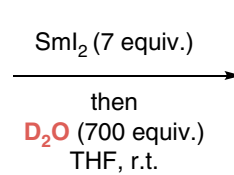

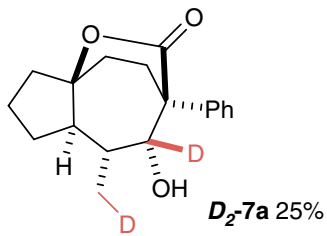

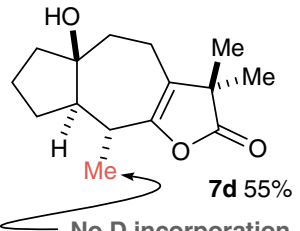

No D incorporation

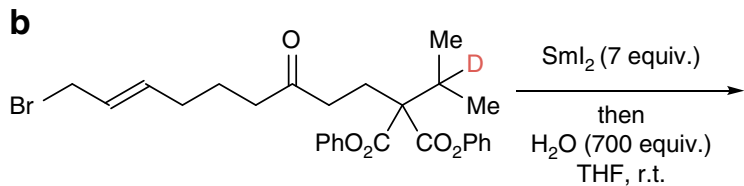

$D-6 \mathrm{~d}$ $(90 \%$ D)

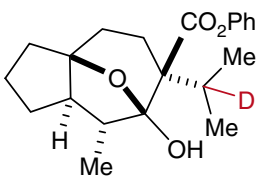
7 p $17 \%$

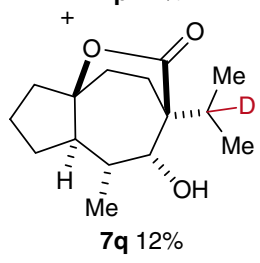

Not observed $7 q 12 \%$

Fig. 6 Mechanistic studies. Mechanistic studies involving the use of a labelled additive and a labelled substrate are shown. $\mathbf{a}, \mathrm{Reaction}$ with $\mathrm{Sml} \mathrm{2}_{2}-\mathrm{D}_{2} \mathrm{O}$ resulted in the formation of deuterated product $\mathbf{D}_{\mathbf{2}} \mathbf{- 7 a}$. This observation supports the operation of the extended SET mechanistic pathway when 1,5-HAT in $\mathbf{V}$ is not possible. Treatment of substrate $\mathbf{6 d}$ with $\mathrm{Sml}_{2}-\mathrm{D}_{2} \mathrm{O}$ resulted in the formation of non-deuterated product $\mathbf{7 d}$. This reaction outcome supports the operation of a mechanism involving activation of a secondary alkyl group by 1,5-HAT. b, Use of deuterated substrate $\mathbf{D}-\mathbf{6} \mathbf{d}$ in the reaction resulted in the formation of products $\mathbf{7 p}$ and $\mathbf{7 q}$ (product $\mathbf{D}-\mathbf{7 d}$ was not formed): The presence of deuterium in $\mathbf{D}-\mathbf{6 d}$ blocked 1,5 -HAT and redirected folding

intermediate (cf. V) is not quenched by reduction/protonation but is instead quenched by intramolecular HAT.

To provide further support for 1,5-HAT, labelled substrate $\boldsymbol{D}$ 6d was prepared in which the tertiary hydrogen atom implicated in the HAT processes was exchanged for deuterium. Notably, upon treatment with $\mathrm{SmI}_{2}$ and $\mathrm{H}_{2} \mathrm{O}$, cyclisation cascade products $7 \mathbf{p}$ and $7 \mathbf{q}(29 \%)$, rather than labelled $\mathbf{7 d}$, were isolated. As 1,5deuterium transfer is known to be slower than $1,5-\mathrm{HAT}^{53}$, the deuterium atom in $\mathbf{D}$-6d blocks the final stages of the folding cascade and redirects the process. It is known that deuterium can be used as a 'protecting group' to prevent HAT processes and thus the outcome of the reaction of $\mathbf{D}$-6d lends further support to the importance of such a process in the folding cascades ${ }^{54}$.

\section{Discussion}

In summary, we have developed a folding cascade approach to complex polycyclic architectures bearing multiple stereocentres mediated by reductive SET from $\mathrm{SmI}_{2}$. The simple, linear substrates for folding are prepared in a modular, two-step synthesis and straightforward variation of substrate structure leads to three different folding pathways that deliver very different molecular architectures. Two of the folding pathways involve the use of 1,5HAT to activate and functionalise otherwise inert secondary alkyl and benzylic groups in the substrates. Notably, 1,5-HAT is scarcely seen in the chemistry of $\mathrm{SmI}_{2}$ and rarely exploited and our studies suggest that the incorporation of 1,5-HAT in synthetic methods involving the SET reagent $\mathrm{SmI}_{2}$ can now be considered. In the context of diversity-oriented synthesis, the incorporation of 1,5-HAT processes in radical carbon-carbon bond-forming cascades has the potential to generate complexity and unlock access to diverse molecular structures in the search for new bioactive natural product-like compounds.

\section{Methods}

General experimental and characterization. Supplementary Figures 1-82 for the nuclear magnetic resonance spectra, Supplementary Tables 1-6 for X-ray crystallographic data, and Supplementary Methods giving full experimental details and the characterization of compounds are given in the Supplementary Information.

Cascade involving an extended SET pathway. To a solution of $\mathrm{SmI}_{2}(9.00 \mathrm{~mL}$, $0.90 \mathrm{mmol}, 0.1 \mathrm{M}$ in tetrahydrofuran (THF)), under nitrogen, $\delta$-keto ester $\mathbf{6} \mathbf{a}^{\prime}-\mathbf{c}^{\prime}$ or 6a-c $(0.11 \mathrm{mmoL})$ in THF $(0.70 \mathrm{~mL})$ was added dropwise and the reaction mixture stirred for $14 \mathrm{~h}$ at room temperature. After that time, degassed $\mathrm{H}_{2} \mathrm{O}(2.20 \mathrm{~mL}, 122$ $\mathrm{mmoL}$ ) was added and the reaction was stirred at the same temperature for $24 \mathrm{~h}$ before being quenched with air, followed by saturated aqueous Rochelle's salt and saturated aqueous sodium thiosulphate. The aqueous layer was extracted with $\mathrm{Et}_{2} \mathrm{O}$ $(3 \times 15 \mathrm{~mL})$ and the combined organic layers were washed with brine $(15 \mathrm{~mL})$, dried over $\mathrm{MgSO}_{4}$, concentrated in vacuo, and purified by column chromatography eluting with EtOAc/hexane (5:95), to give compound $7 \mathbf{a}^{\prime}-\mathbf{c}^{\prime}$ or $\mathbf{7 a - c}$.

Cascade involving 1,5-HAT and activation of a secondary alkyl group. To a solution of $\mathrm{SmI}_{2}$ ( $7.00 \mathrm{~mL}, 0.70 \mathrm{mmoL} 0.1 \mathrm{M}$ in THF), under nitrogen, malonate $\mathbf{6 d}-\mathbf{j}(0.10 \mathrm{mmoL})$ in THF $(0.70 \mathrm{~mL})$ was added dropwise and the reaction mixture stirred for $14 \mathrm{~h}$ at room temperature. After that time, degassed $\mathrm{H}_{2} \mathrm{O}(1.26 \mathrm{~mL}, 70.0$ mmol) was added and the reaction was stirred at the same temperature for $24 \mathrm{~h}$ before being quenched with air, followed by saturated aqueous Rochelle's salt and saturated aqueous sodium thiosulfate. The aqueous layer was extracted with $\mathrm{Et}_{2} \mathrm{O}$ $(3 \times 15 \mathrm{~mL})$ and the combined organic layers were washed with brine $(15 \mathrm{~mL})$, dried over $\mathrm{MgSO}_{4}$, concentrated in vacuo, and purified by column chromatography eluting with EtOAc/hexane (5:95), to give compound $\mathbf{7} \mathbf{d}-\mathbf{j}$.

Cascade involving 1,5-HAT and activation of a benzylic group. To a solution of $\mathrm{SmI}_{2}(7.00 \mathrm{~mL}, 0.70 \mathrm{mmoL}, 0.1 \mathrm{M}$ in THF), malonate $\mathbf{6 k - q}(0.10 \mathrm{mmoL})$ in THF 
$(0.70 \mathrm{~mL})$ was added dropwise under nitrogen and stirred for $14 \mathrm{~h}$ at room temperature. After that time, degassed $\mathrm{MeOH}(340 \mu \mathrm{L}, 8.4 \mathrm{mmol})$ was added and the reaction was stirred at the same temperature for $48 \mathrm{~h}$ before being quenched with air followed by saturated aqueous Rochelle's salt and saturated aqueous sodium thiosulfate. The aqueous layer was extracted with $\mathrm{Et}_{2} \mathrm{O}(3 \times 15 \mathrm{~mL})$ and the combined organic layers were washed with brine $(15 \mathrm{~mL})$, dried over $\mathrm{MgSO}_{4}$, concentrated in vacuo, and purified by column chromatography eluting with $\mathrm{Et}_{2} \mathrm{O}$ / pentane (20:80), to give compound $7 \mathbf{k}-\mathbf{q}$.

\section{Data availability}

The X-ray crystallographic coordinates for $7 \mathbf{a}^{\prime}, 7 \mathbf{d}^{\prime}, 7 \mathbf{a}, 7 \mathbf{d}, \mathbf{7 f}$, and $7 \mathbf{k}$ have been deposited at the Cambridge Crystallographic Data Centre (CCDC) under deposition numbers CCDC 1825583 and 1825585-89. These data can be obtained free of charge from the CCDC via www.ccdc.cam.ac.uk/data_request/cif. The authors declare that all other data supporting the findings of this study are available within the article and its Supplementary Information file.

Received: 14 May 2018 Accepted: 16 October 2018

Published online: 15 November 2018

\section{References}

1. Schreiber, S. L. Target-oriented and diversity-oriented organic synthesis in drug discovery. Science 287, 1964-1969 (2000).

2. Tan, D. S. Diversity-oriented synthesis: exploring the intersections between chemistry and biology. Nat. Chem. Biol. 1, 74-84 (2005).

3. O' Connor, C. J., Beckmann, H. S. G. \& Spring, D. R. Diversity-oriented synthesis: producing chemical tools for dissecting biology. Chem. Soc. Rev. 41, 4444-4456 (2012).

4. Kato, N. et al. Diversity-oriented synthesis yields novel multistage antimalarial inhibitors. Nature 538, 344-349 (2016).

5. Galloway, W. R. J. D., Isidro-Llobet, A. \& Spring, D. R. Diversity-oriented synthesis as a tool for the discovery of novel biologically active small molecules. Nat. Commun. 1, 80 (2010).

6. Ibbeson, B. M. et al. Diversity-oriented synthesis as a tool for identifying new modulators of mitosis. Nat. Commun. 5, 3155 (2014).

7. Karageorgis, G., Warriner, S. \& Nelson, A. Efficient discovery of bioactive scaffolds by activity-directed synthesis. Nat. Chem. 6, 872-876 (2014).

8. Wang, Y. et al. Diversity-oriented synthesis as a strategy for fragment evolution against GSK3 3 . ACS Med. Chem. Lett. 7, 852-856 (2016)

9. Thomas, G. L. et al. Anti-MRSA agent discovery using diversity-oriented synthesis. Angew. Chem. Int. Ed. 47, 2808-2812 (2008).

10. Patil, N. T., Shinde, V. S. \& Sridhar, B. Relay catalytic branching cascade: a technique to access diverse molecular scaffolds. Angew. Chem. Int. Ed. 52, 2251-2255 (2013).

11. Grossmann, A., Bartlett, S., Janecek, M., Hodgkinson, J. T. \& Spring, D. R. Diversity-oriented synthesis of drug-like macrocyclic scaffolds using an orthogonal organo- and metal catalysis strategy. Angew. Chem. Int. Ed. 53, 13093-13097 (2014).

12. Mizoguchi, H., Oikawa, H. \& Oguri, H. Biogenetically inspired synthesis and skeletal diversification of indole alkaloids. Nat. Chem. 6, 57-64 (2014).

13. Echemendía, R. et al. Highly stereoselective synthesis of natural-product-like hybrids by an organocatalytic/multicomponent reaction sequence. Angew. Chem. Int. Ed. 54, 7621-7625 (2015).

14. Lachkar, D. et al. Unified biomimetic assembly of voacalgine A and bipleiophylline via divergent oxidative couplings. Nat. Chem. 9, 793-798 (2017).

15. Oliver, D. W. \& Malan, S. F. Medicinal chemistry of polycyclic cage compounds in drug discovery research. Med. Chem. Res. 17, 137-151 (2008).

16. Stockdale, T. P. \& Williams, C. M. Pharmaceuticals that contain polycyclic hydrocarbon scaffolds. Chem. Soc. Rev. 44, 7737-7763 (2015).

17. Lovering, F., Bikker, J. \& Humblet, C. Escape from flatland: increasing saturation as an approach to improving clinical success. J. Med. Chem. 52, 6752-6756 (2009).

18. Burke, M. D. \& Schreiber, S. L. A planning strategy for diversity-oriented synthesis. Angew. Chem. Int. Ed. 43, 46-58 (2004).

19. Nicolaou, K. C., Edmonds, D. J. \& Bulger, P. G. Cascade reactions in total synthesis. Angew. Chem. Int. Ed. 45, 7134-7186 (2006)

20. Morton, D., Leach, S., Cordier, C., Warriner, S. \& Nelson, A. Synthesis of natural-product-like molecules with over eighty distinct scaffolds. Angew. Chem. Int. Ed. 48, 104-109 (2009).

21. Liu, W., Khedkar, V., Baskar, B., Schürmann, M. \& Kumar, K. Branching cascades: a concise synthetic strategy targeting diverse and complex molecular frameworks. Angew. Chem. Int. Ed. 50, 6900-6905 (2011).

22. Garcia-Castro, M. et al. De novo branching cascades for structural and functional diversity in small molecules. Nat. Commun. 6, 6516 (2015).
23. Burke, M. D., Berger, E. M. \& Schreiber, S. L. Generating diverse skeletons of small molecules combinatorially. Science 302, 613-618 (2003).

24. Plesniak, M. P., Huang, H.-M. \& Procter, D. J. Radical cascade reactions triggered by single electron transfer. Nat. Rev. Chem. 1, 77 (2017).

25. Kern, N., Plesniak, M. P., McDouall, J. J. W. \& Procter, D. J. Enantioselective cyclizations and cyclization cascades of samarium ketyl radicals. Nat. Chem. $\mathbf{9}$, 1198-1204 (2017)

26. Keylor, M. H. et al. Synthesis of resveratrol tetramers via a stereoconvergent radical equilibrium. Science 354, 1260-1265 (2016).

27. Chu, J. C. K. \& Rovis, T. Amide-directed photoredox-catalysed C-C bond formation at unactivated $\mathrm{sp}^{3} \mathrm{C}-\mathrm{H}$ bonds. Nature 539, 272-275 (2016).

28. Choi, G. J., Zhu, Q., Miller, D. C., Gu, C. J. \& Knowles, R. R. Catalytic alkylation of remote $\mathrm{C}-\mathrm{H}$ bonds enabled by proton-coupled electron transfer. Nature 539, 268-271 (2016)

29. Dauncey, E. M., Morcillo, S. P., Douglas, J. J., Sheikh, N. S. \& Leonori, D. Photoinduced remote functionalisations by iminyl radical promoted $\mathrm{C}-\mathrm{C}$ and C-H bond cleavage cascades. Angew. Chem. Int. Ed. 57, 744-748 (2018).

30. Shu, W., Lorente, A., Gómez-Bengoa, E. \& Nevado, C. Expeditious diastereoselective synthesis of elaborated ketones via remote $\mathrm{Csp}^{3}-\mathrm{H}$ functionalization. Nat. Commun. 8, 13832 (2017).

31. Yamago, S., Miyazoe, H., Nakayama, T., Miyoshi, M. \& Yoshida, J. A diversity-oriented synthesis of $\alpha$-amino acid derivatives by a silyltelluridemediated radical coupling reaction of imines and isonitriles. Angew. Chem. Int. Ed. 42, 117-120 (2003).

32. Li, L. et al. Radical aryl migration enables diversity-oriented synthesis of structurally diverse medium/macro- or bridged-rings. Nat. Commun. 7, 13852 (2016).

33. Kuznetsov, D. M. \& Kutateladze, A. G. Step-economical photoassisted diversity-oriented synthesis: sustaining cascade photoreactions in oxalyl anilides to access complex polyheterocyclic molecular architectures. J. Am. Chem. Soc. 139, 16584-16590 (2017).

34. Wang, X. et al. A radical cascade enabling collective syntheses of natural products. Chem 2, 803-816 (2017).

35. Szostak, M., Fazakerley, N. J., Parmar, D. \& Procter, D. J. Cross-coupling reactions using samarium(II) iodide. Chem. Rev. 114, 5959-6039 (2014).

36. Molander, G. A. Application of lanthanide reagents in organic synthesis. Chem. Rev. 92, 29-68 (1992).

37. Kagan, H. B. Twenty-five years of organic chemistry with diiodosamarium: an overview. Tetrahedron 59, 10351-10372 (2003).

38. Beemelmanns, C. \& Reissig, H.-U. Samarium diiodide induced ketyl-(het) arene cyclisations towards novel N-heterocycles. Chem. Soc. Rev. 40, 2199-2210 (2011).

39. Murakami, M., Hayashi, M. \& Ito, Y. Generation and alkylation of carbanions a to the nitrogen of amines by a new metallation procedure. J. Org. Chem. 57, 793-794 (1992).

40. Hölemann, A. \& Reissig, H.-U. Samarium diiodide-induced couplings of carbonyl compounds with methoxyallene leading to 4-hydroxy 1-enol ethers. Org. Lett. 5, 1463-1466 (2003).

41. Hölemann, A. \& Reißig, H.-U. Regioselective samarium diiodide induced couplings of carbonyl compounds with 1,3-diphenylallene and alkoxyallenes: a new route to 4-hydroxy-1-enol ethers. Chem. Eur. J. 10, 5493-5506 (2004).

42. Just-Baringo, X. \& Procter, D. J. Sm(II)-mediated electron transfer to carboxylic acid derivatives: development of complexity generating cascades. ACC Chem. Res. 48, 1263-1275 (2015).

43. Kamochi, Y. \& Kudo, T. Novel reduction of carboxylic acids, esters, amides and nitriles using samarium diiodide in the presence of water. Chem. Lett. 22, 1495-1498 (1993).

44. Parmar, D. et al. Reductive cyclization cascades of lactones using $\mathrm{SmI}_{2}-\mathrm{H}_{2} \mathrm{O}$. J. Am. Chem. Soc. 133, 2418-2420 (2011).

45. Hasegawa, E. \& Curran, D. P. Rate constants for the reactions of primary alkyl radicals with $\mathrm{SmI}_{2}$ in THF/HMPA. Tetrahedron Lett. 34, 1717-1720 (1993).

46. Karl, C. L., Maas, E. J. \& Reusch, W. Acyl rearrangements in radical reactions. J. Org. Chem. 37, 2834-2840 (1972).

47. Rudkin, I. M., Miller, L. C. \& Procter, D. J. Samarium enolates and their applications in organic synthesis. Organomet. Chem. 34, 19-45 (2008).

48. Chopade, P. R., Prasad, E. \& Flowers, R. A. The role of proton donors in $\mathrm{SmI}_{2}$ mediated ketone reduction: new mechanistic insights. J. Am. Chem. Soc. 126 $44-45$ (2004).

49. Keck, G. E. \& Wager, C. A. The first directed reduction of $\beta$-alkoxy ketones to anti 1,3-diol monoethers: identification of spectator and director alkoxy groups. Org. Lett. 2, 2307-2309 (2000).

50. Hutton, T. K., Muir, K. W. \& Procter, D. J. Switching between novel samarium (II)-mediated cyclizations by a simple change in alcohol cosolvent. Org. Lett. 5, 4811-4814 (2003).

51. Szostak, M., Spain, M., Choquette, K. A., Flowers, R. A. II \& Procter, D. J. Substrate-directable electron transfer reactions. dramatic rate enhancement in the chemoselective reduction of cyclic esters using $\mathrm{SmI}_{2}-\mathrm{H}_{2} \mathrm{O}$ : mechanism, scope, and synthetic utility. J. Am. Chem. Soc. 135, 15702-15705 (2013). 
52. Szostak, M., Spain, M. \& Procter, D. J. Selective synthesis of a,a-dideuterio alcohols by the reduction of carboxylic acids using $\mathrm{SmI}_{2}$ and $\mathrm{D}_{2} \mathrm{O}$ as deuterium source under SET conditions. Org. Lett. 16, 5052-5055 (2014).

53. DeZutter, C. B., Horner, J. H. \& Newcomb, M. Rate constants for 1,5- and 1,6hydrogen atom transfer reactions of mono-, di-, and tri-aryl-substituted donors, models for hydrogen atom transfers in polyunsaturated fatty acid radicals. J. Phys. Chem. A 112, 1891-1896 (2008).

54. Wood, M. E., Bissiriou, S., Lowe, C. \& Windeatt, K. M. Investigations into the effectiveness of deuterium as a 'protecting group' for $\mathrm{C}-\mathrm{H}$ bonds in radical reactions involving hydrogen atom transfer. Org. Biomol. Chem. 6, 3048-3051 (2008).

\section{Acknowledgements}

This work was partially supported by the EPSRC (DTA Studentship to M.P.P.; Postdoctoral Fellowship to to X.J.-B. (EP/L00125X/1); Established Career Fellowship to D.J. P. (EP/M005062/1)), CONACyT, México (PhD Scholarship No. 510789 to M.H.G.-C.), and Erasmus+ (placement for P.L.).

\section{Author contributions}

M.P.P. and D.J.P. conceived the study and co-wrote the manuscript. M.P.P. designed and performed experiments, and M.H.G.-C., P.L., and X.J.-B. performed experiments.

\section{Additional information}

Supplementary Information accompanies this paper at https://doi.org/10.1038/s41467018-07194-x
Competing interests: The authors declare no competing interests.

Reprints and permission information is available online at http://npg.nature.com/ reprintsandpermissions/

Publisher's note: Springer Nature remains neutral with regard to jurisdictional claims in published maps and institutional affiliations.

\section{(c) (1)}

Open Access This article is licensed under a Creative Commons Attribution 4.0 International License, which permits use, sharing, adaptation, distribution and reproduction in any medium or format, as long as you give appropriate credit to the original author(s) and the source, provide a link to the Creative Commons license, and indicate if changes were made. The images or other third party material in this article are included in the article's Creative Commons license, unless indicated otherwise in a credit line to the material. If material is not included in the article's Creative Commons license and your intended use is not permitted by statutory regulation or exceeds the permitted use, you will need to obtain permission directly from the copyright holder. To view a copy of this license, visit http://creativecommons.org/ licenses/by/4.0/.

(C) The Author(s) 2018 